\title{
Induction of differentiation-specific miRNAs in TPA-induced myeloid leukemia cells through MEK/ERK activation
}

\author{
JING WANG ${ }^{1}$, LIJUN LIU ${ }^{2}$, LAN XIE $^{1}$, GUANGXIN XIANG $^{3}$ and YUXIANG ZHOU ${ }^{1,3,4}$ \\ ${ }^{1}$ Medical Systems Biology Research Center, School of Medicine, Tsinghua University, Beijing 100084; \\ ${ }^{2}$ Reproductive Medicine Center, The First Hospital of Lanzhou University, Lanzhou, Gansu 730000; \\ ${ }^{3}$ National Engineering Research Center for Beijing Biochip Technology, Beijing 102206; ${ }^{4}$ The State Key \\ Laboratory of Biomembrane and Membrane Biotechnology, Tsinghua University, Beijing 100084, P.R. China
}

Received August 7, 2012; Accepted September 21, 2012

DOI: 10.3892/ijmm.2012.1191

\begin{abstract}
CellularmicroRNAs (miRNAs) are pivotal regulators involved in various biological processes through the post-transcriptional regulation of gene expression. Signaling pathways are extensively activated during 12-O-Tetradecanoylphorbol13-acetate (TPA)-induced differentiation of human leukemia cells, but the modulation of miRNA expression and processing in this context has yet to be fully explored. In this study, we comprehensively analyzed 10 miRNAs that are consistently upregulated during TPA-induced differentiation of various leukemia cell lines by employing microarray technology. The upregulation of these miRNAs was further verified by quantitative RT-PCR, and, markedly, a subset of the miRNAs was found to be induced via the MEK/ERK signaling pathway using TPA and specific pharmacological inhibitors. Moreover, immunoblotting and quantitative RT-PCR analysis demonstrated that the expression levels of key miRNA processing machineries (i.e., Drosha, Dicer, Ago1 and Ago2) were not induced in this context, but the transcription of the miRNA products was triggered by MEK/ERK activation. Therefore, we identified the unique miRNAs that respond to TPA treatment in leukemia cells and demonstrated the essential role of the MEK/ERK signaling pathway in the induction of these miRNA transcripts.
\end{abstract}

\section{Introduction}

Dysregulation of hematopoietic cellular differentiation contributes to leukemogenesis (1). The use of differentiation agents can force malignant cells to undergo terminal differentiation, which is viewed as a promising and revolutionary approach for the therapy of leukemia diseases (2). The drug

Correspondence to: Professor Yuxiang Zhou, Medical Systems Biology Research Center, School of Medicine, Tsinghua University, Beijing 100084, P.R. China

E-mail: zhouyx@mail.tsinghua.edu.cn

Key words: myeloid leukemia, 12-O-Tetradecanoylphorbol-13-acetate, microRNA, microRNA biogenesis machinery, MEK/ERK signaling
12-O-Tetradecanoylphorbol-13-acetate (TPA) is a phase I clinical therapeutic agent for patients with relapsed/refractory myelocytic leukemia due to its capacity to induce differentiation or apoptosis in malignant cells (3). Further molecular and genetic analyses of the effects of TPA could promote a better understanding of its mechanisms contributing to differentiation and therapeutic response.

A new class of small non-coding RNA molecules known as microRNAs (miRNAs) has introduced a whole new layer of gene regulation in eukaryotes (4). The miRNAs are transcribed by RNA polymerase II as large primary non-coding transcripts or from regions of a known gene, and the transcribed primary miRNAs are processed through the concerted actions of biogenesis machineries, including their sequential cleavage, export, and incorporation into the RNA-induced silencing complex (RISC) to mediate the expression of target genes $(5,6)$. miRNA expression profiling in TPA-treated leukemia cells has previously been performed in several studies (7-9). However, considering the number of miRNAs that exist in cells and the various types of myeloid leukemia, additional differentiationrelated miRNAs need to be identified (10). Moreover, miRNA expression profiling should be conducted and compared among various leukemia cell lines to identify the specifically induced miRNAs that respond to TPA treatment.

The differentiation of leukemia cells induced by TPA is highly dependent on the involvement of multiple signaling pathways, including the mitogen-activated protein kinase (MAPK) pathways, involving MEK/ERK/MAP kinase and c-Jun NH2-terminal kinase, as well as MAPK-independent pathways such as the PI3K pathway (11). These signaling pathways are responsible for appropriately mediating gene transcription with respect to cellular behavior (12). To better understand the mechanism of TPA action, it is useful to investigate which pathways are activated and how they mediate the induction of miRNAs in response to TPA treatment in leukemia cells.

Here, we present the results of a microarray-based screen for miRNAs that respond to TPA treatment in various leukemia cell lines. We identified a series of specific differentiation-induced miRNAs and analyzed their responses to signal transduction by using pharmacological inhibitors, showing the essential role of MEK/ERK signaling in miRNA induction. Moreover, the 
regulation of both miRNA biogenesis machineries and primary transcripts was analyzed in the same context, revealing the major mechanisms for the induction of miRNA products.

\section{Materials and methods}

Cell culture and reagents. The NB4, HL-60, K562 and U937 cell lines were obtained from the American Type Culture Collection (ATCC, Richmond, MD, USA) and cultured in RPMI-1640 medium (Gibco, Grand Island, NY, USA) supplemented with $10 \%$ fetal bovine serum (PAA Laboratories Pty Ltd, Morningside, Australia), $2 \mathrm{mM} \mathrm{L-glutamine} \mathrm{and} \mathrm{antibi-}$ otics in a humidified incubator containing $5 \% \mathrm{CO}_{2}$ at $37^{\circ} \mathrm{C}$. TPA, U0126 and LY294002 were purchased from Sigma (St. Louis, MO, USA) and dissolved in dimethylsulfoxide (DMSO) according to the supplier's instructions. To induce cell differentiation, TPA was added to the medium to a final concentration of $30 \mathrm{nM}$ (13). To inhibit signal transduction, cells were pretreated with specific inhibitors for $30 \mathrm{~min}$ prior to TPA treatment $(14,15)$. Antibodies against phospho-ERK1/2, ERK1/2, Dicer, Drosha, Ago1, and Ago2 were purchased from Cell Signaling Technology (Danvers, MA, USA). Antibodies against phospho-Akt, Akt and $\beta$-actin were obtained from Santa Cruz Biotechnology, Inc. (Santa Cruz, CA, USA). The horseradish peroxidase (HRP)-conjugated secondary antibodies were also obtained from Santa Cruz Biotechnology, Inc.

Assessment of surface antigen expression. Surface antigen expression was measured by flow cytometry according to our previously published protocol (13). Briefly, cells were harvested at the indicated times, washed twice with PBS, and then incubated with $20 \mu 1$ FITC-labeled anti-CD14 antibody working solution (eBioscience, San Diego, CA, USA) for $30 \mathrm{~min}$ at $4^{\circ} \mathrm{C}$ in the dark. For every sample, separate aliquots of cells were also incubated with isotype antibody controls (eBioscience) to determine non-specific staining. Following incubation, cells were washed twice with PBS and analyzed by flow cytometry with a $488 \mathrm{~nm}$ argon laser. For each sample, a total of 10,000 cells were analyzed.

Assessment of cell cycle. Cell cycle was profiled by flow cytometry as previously described (14). Briefly, cells were harvested and fixed in $70 \%$ ethanol at $4^{\circ} \mathrm{C}$ overnight. After washing with PBS, the fixed cells were re-suspended in PBS containing $100 \mu \mathrm{g} / \mathrm{ml}$ RNase A and incubated for $30 \mathrm{~min}$ at $37^{\circ} \mathrm{C}$. Finally, the cells were collected by centrifugation and incubated in PBS containing $50 \mu \mathrm{g} / \mathrm{ml}$ of propidium iodide (PI) (Sigma) for $20 \mathrm{~min}$ in the dark, and then analyzed using flow cytometry with a $488 \mathrm{~nm}$ argon laser. A minimum of 20,000 cells was analyzed for each sample.

miRNA microarray analysis. For miRNA microarray analysis, total RNA was extracted using TRIzol Reagent (Invitrogen, Carlsbad, CA, USA) according to the manufacturer's instructions. CapitalBio Mammalian miRNA Array V3.0 (CapitalBio, Beijing, China) microarrays were then probed, which include 924 probes for human, mouse and rat miRNAs. The microarray analyses were performed according to our previously published protocols $(13,16)$. Significance Analysis of Microarrays (SAM; Stanford University, CA, USA) software was used to determine the differentially expressed miRNAs, and the selection criteria included a fold change $\geq 1.5$ or $\leq 0.65$, a q-value $\leq 5 \%$, and a SAM score $>2$ or $<-2$ (17). The entire dataset described here is available from the Gene Expression Omnibus (GEO, http://www. ncbi.nlm.nih.gov/geo) through accession number GSE33537.

Quantitative real-time PCR analysis. To quantify the mature miRNAs, stem-loop RT-PCR assays were performed according to the method of Chen et al (18); snRNA U6 was used as an internal standard. To quantify the pri-miRNAs, total RNA was subjected to DNase I treatment and reversetranscribed into cDNA with M-MLV (Promega, Wisconsin, WI, USA) (19). Following reverse transcription, PCR reactions were performed using a SuperGreen quantitative PCR kit II (CapitalBio) according to the manufacturer's instructions. ACTB was used as an internal standard. Relative expression levels were calculated using the formula: Q.rel. $=2^{-\Delta \mathrm{CT}}$, where $\Delta \mathrm{CT}=$ average CT test gene - average CT internal standard, and $\mathrm{CT}$ is the cycle threshold. All primers used for quantitative RT-PCR are listed in Table I.

Immunoblotting analysis. The cells were quickly lysed on ice using lysis buffer $(20 \mathrm{mM}$ Tris, $\mathrm{pH} 7.5,150 \mathrm{mM} \mathrm{NaCl}, 1 \%$ Triton X-100, 10\% glycerol, $2 \mathrm{mM}$ EDTA, $1 \mathrm{mM} \mathrm{Na}_{3} \mathrm{VO}_{4}$, and protease inhibitors) and clarified by centrifugation at $14,000 \mathrm{rpm}$ for $10 \mathrm{~min}$; the supernatants represented the cellular protein extracts. Equal quantities of protein extracts were resolved by SDS-PAGE and transferred to nitrocellulose membranes. The membranes were blocked in TBST $(10 \mathrm{mM}$ Tris $\mathrm{pH}$ 7.6, $150 \mathrm{mM} \mathrm{NaCl}$, and $1 \%$ Tween-20) containing 5\% low-fat milk for $1 \mathrm{~h}$. Subsequently, the membranes were incubated with primary antibody dilutions at $4^{\circ} \mathrm{C}$ overnight, followed by three washes with TBST at room temperature. The membranes were then incubated with secondary antibody dilutions for $1 \mathrm{~h}$ at room temperature, followed by four washes with TBST. The enhanced chemiluminescence (ECL) system (Pierce, Rockford, IL, USA) was used to detect reactive proteins.

Statistical analysis. Experiments were performed in duplicate or triplicate and independently repeated at least three times. Data are presented as the means \pm standard deviation (SD). Statistical significance was determined using two-tailed t-tests, and $\mathrm{P}<0.05$ was considered to indicate statistically significant differences.

\section{Results}

Analysis of the miRNA expression pattern of myeloid leukemia cell lines following TPA treatment. NB4 (acute promyelocytic leukemia), HL-60 (acute myeloblastic leukemia), U937 (monoblastic leukemia), and K562 (chronic myelogenous leukemia) cell lines are the typical models for studies of human leukemia cell differentiation in vitro. The differentiation of these cells induced by TPA was determined by assessing cell morphology and the expression of a differentiation marker. After TPA treatment, the cells spread and adhered to the culture dishes, and some cells displayed pseudopod-like protrusions (Fig. 1A). A significant increase in CD14 expression was also observed in leukemia cells treated with TPA (Fig. 1B). These results suggest that cellular differentiation was induced. 
Table I. PCR primers designed for the amplification of the investigated microRNAs and primary transcripts.

\begin{tabular}{lll}
\hline Gene & \multicolumn{1}{c}{ Forward primer (5'-3') } & Reverse primer (5'-3') \\
\hline hsa-miR-21 & GCCGCTAGCTTATCAGACTGATGT & GTGCAGGGTCCGAGGT \\
hsa-miR-22 & CGAAGCTGCCAGTTGAAGAA & GTGCAGGGTCCGAGGT \\
hsa-miR-23a & GATATCACATTGCCAGGGATT & GTGCAGGGTCCGAGGT \\
hsa-miR-24 & GGTGGCTCAGTTCAGCAGGA & GTGCAGGGTCCGAGGT \\
hsa-miR-23b & GCATCACATTGCCAGGGATT & GTGCAGGGTCCGAGGT \\
hsa-miR-29a & CGTAGCACCATCTGAAATCGG & GTGCAGGGTCCGAGGT \\
hsa-miR-29b & GCATAGCACCATTTGAAATAGT & GTGCAGGGTCCGAGGT \\
hsa-miR-146b & CGGCTGAGAACTGAATTCCATAG & GTGCAGGGTCCGAGGT \\
hsa-miR-638 & AGGGATCGCGGGCGG & GTGCAGGGTCCGAGGT \\
SnRNA U6 & CTCGCTTCGGCAGCACA & AACGCTTCACGAATTTGCGT \\
Pri-miR-21 & TTTTGTTTTGCTTGGGAGGA & AGCAGACAGTCAGGCAGGAT \\
Pri-miR-22 & GCAGAAAGCCTTGGGTTG & CGAACAGCAGGGTGGATGA \\
Pri-23a-24-2 & TCACCCCTGTGCCACG & CAAACCAACTGTGTTTCAGCT \\
Pri-miR-146b & GAGCAGCGTCCAGGCTG & CCGGGCACCAGAACTGAGT \\
ACTB & CATGTACGTTGCTATCCAGGC & CTCCTTAATGTCACGCACGAT
\end{tabular}

Table II. Significantly upregulated miRNAs obtained by microarray analysis.

Cell lines $\quad$ miRNAs (fold changes $^{\mathrm{a}} \geq 1.5$-folds; $\mathrm{q}$-value ${ }^{\mathrm{b}} \leq 0.05$ )

HL-60 hsa-miR-21, hsa-miR-22, hsa-miR-146a, hsa-miR-27a, hsa-miR-222, hsa-miR-27b, hsa-miR-24, hsa-miR-146b-5p, hsa-miR-23b, hsa-miR-29a, hsa-miR-23a, hsa-miR-221, hsa-miR-509-3p, hsa-miR-17, hsa-miR-29b, PREDICTED_MIR191, hsa-miR-638

NB4 hsa-miR-146a, hsa-miR-222, hsa-miR-22, hsa-miR-23b, hsa-miR-146b-5p, hsa-miR-221, hsa-miR-638, hsa-miR-21, PREDICTED_MIR191, hsa-miR-23a, hsa-miR-24, hsa-miR-27b, hsa-miR-663, hsa-miR-509-3p, hsa-miR-155, hsa-miR-124, mmu-miR-193b, hsa-miR-193a-3p, hsa-miR-29b, hsa-let-7g, hsa-miR-29a

U937 hsa-miR-638, hsa-miR-486-3p, hsa-miR-508-5p, PREDICTED_MIR191, hsa-miR-663, hsa-miR-22, hsa-miR-584, hsa-miR-146b-5p, hsa-miR-487b, hsa-miR-888, hsa-miR-21, hsa-miR-23b, hsa-miR-146a, hsa-miR-27b, PREDICTED_MIR255, hsa-miR-222, hsa-miR-381, hsa-miR-24, hsa-miR-658, hsa-miR-221, hsa-miR-509-3p, hsa-miR-27a, hsa-miR-424, hsa-miR-23a, hsa-miR-28-5p, hsa-miR-193a-3p, hsa-miR-29b, hsa-miR-218, hsa-miR-410, hsa-miR-216a, hsa-miR-29a, hsa-miR-26a, PREDICTED_MIR160, hsa-miR-192, hsa-miR-125b

K562 PREDICTED_MIR191, hsa-miR-638, hsa-miR-663, mmu-miR-762, hsa-miR-22, hsa-miR-21, hsa-miR-146b-5p, hsa-miR-24, hsa-miR-29b, hsa-miR-23b, hsa-miR-27a, hsa-miR-29a, hsa-miR-23a, hsa-miR-17, hsa-miR-16

aa Fold changes indicate the expression level of miRNAs in TPA-treated cells compared to the untreated cells; ${ }^{\mathrm{b}} \mathrm{q}$-value indicates the lowest positive false discovery rate (FDR) at which the gene was considered significant.

Using microarrays, the global changes in miRNA expression were analyzed after treatment of the myeloid leukemia cell lines with TPA. The microarray chips contained 924 probes, allowing a survey of 802 mature human, mouse and rat miRNAs after discarding redundant sequences, and a further 122 predicted miRNAs from published data. To reduce individual variability, replicate array analysis used independently treated samples, and a technical replicate was also performed for each sample.

Employing hierarchical cluster analysis, the global expression patterns of miRNAs in the four leukemia cell lines were obtained (Fig. 1C). Through SAM statistics (17), significantly regulated miRNAs in differentiated cells were identified. TPA induction resulted in the upregulation of 21 miRNAs in the NB 4 cell line, 17 miRNAs in the HL-60 cell line, 15 miRNAs in the K562 cell line, and 35 miRNAs in the U937 cell line (Table II). To identify specific differentiation-induced miRNAs, the overlaps of upregulated miRNAs were evaluated between these four myeloid leukemia lines (20). The cell lines each exhibited characteristic miRNA profiling due to their different origins. However, 10 unique miRNAs were consistently induced in all four leukemia cell lines after exposure 
A

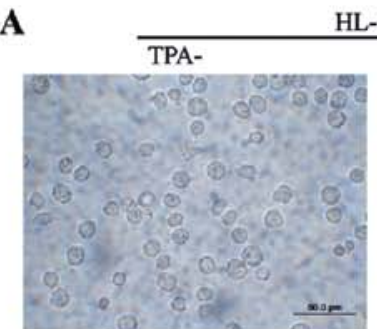

HL-60

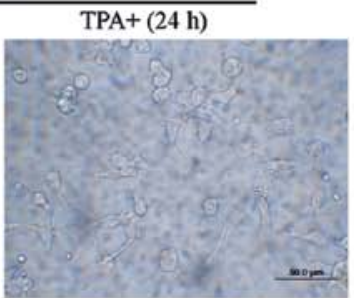

U937
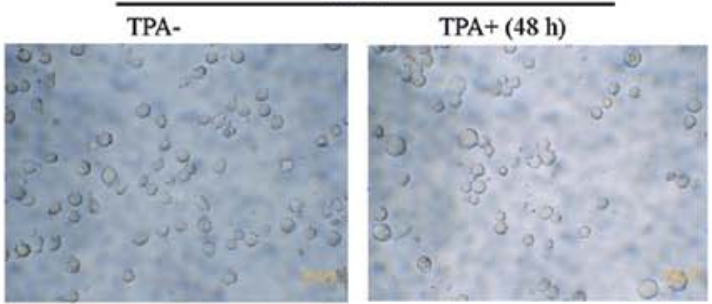

B

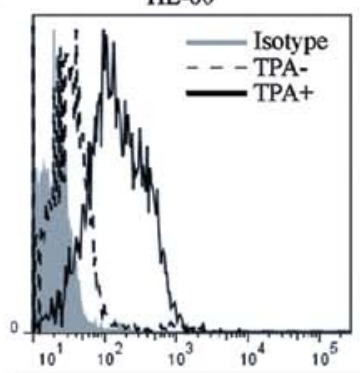

NB4

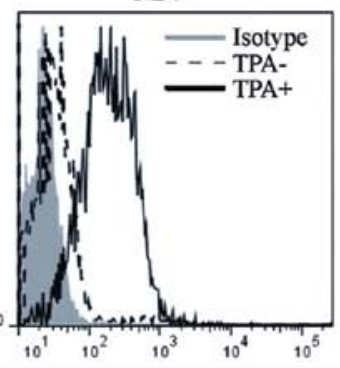

\begin{tabular}{ll}
\multicolumn{2}{c}{ NB4 } \\
\hline TPA- & TPA $+(48 \mathrm{~h})$
\end{tabular}
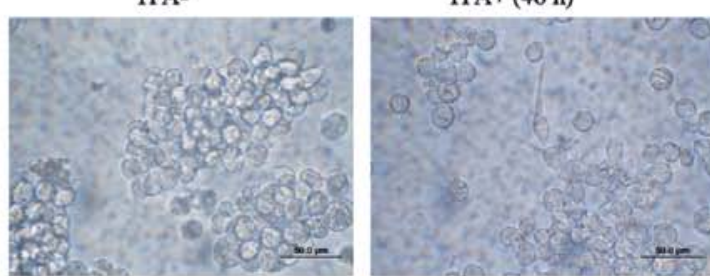

K562

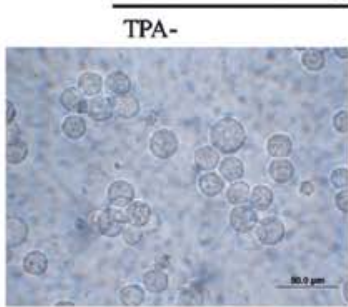

U937

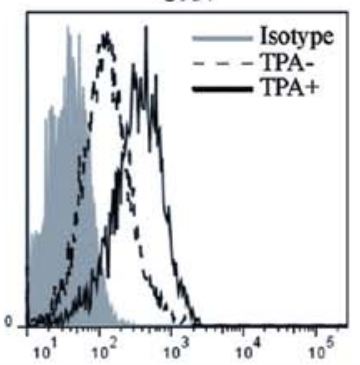

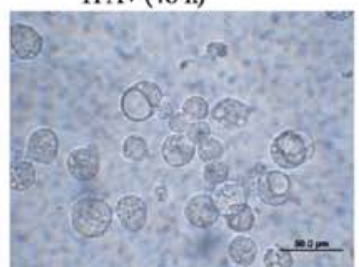

K562

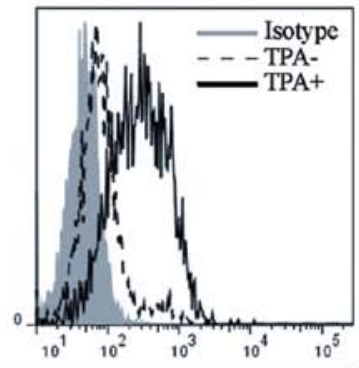

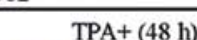

FITC-A

C
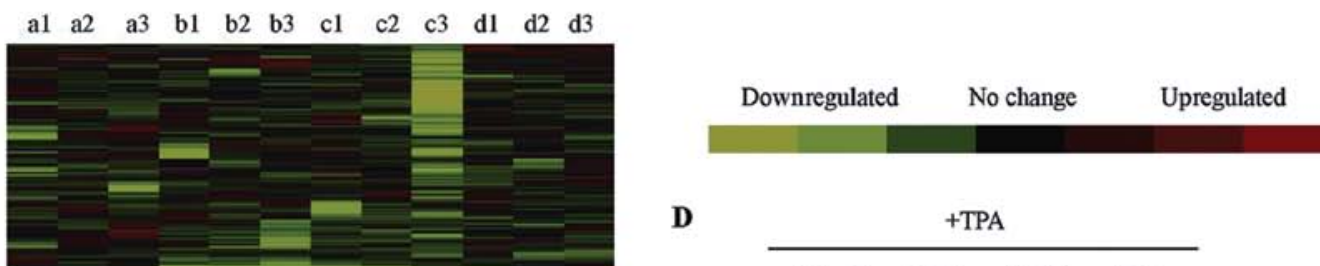

D

+TPA
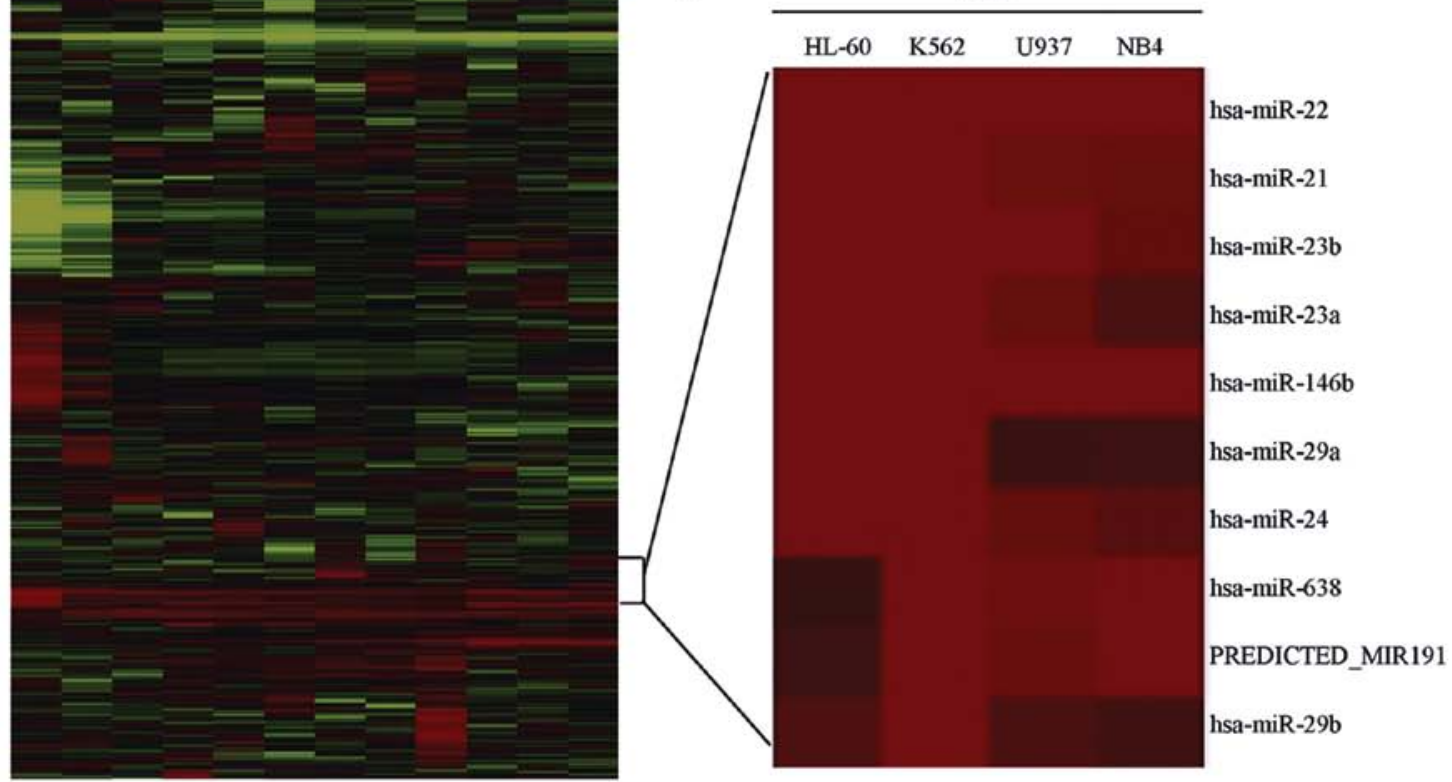

Figure 1. Microarray profiling of miRNAs in myeloid leukemia cell lines treated with TPA. (A) Morphological changes of leukemia cell lines exposed to $30 \mathrm{nM}$ TPA (scale bar, $50 \mu \mathrm{m}$ ). (B) Flow cytometric analysis of CD14 expression in leukemia cell lines treated with TPA. (C) Hierarchical clustering showing the expression patterns of 924 mature miRNAs in four myeloid leukemia cell lines treated with TPA. Samples are labeled a-d: a1-a3, HL-60 (TPA) vs. HL-60 (normal); b1-b3, K562 (TPA) vs. K562 (normal); c1-c3, NB4 (TPA) vs. NB4 (normal); and d1-d3, U937 (TPA) vs. U937 (normal). Biologically independent triplicates are indicated by 1-3. The color bar indicates the expression changes of miRNAs: red represents upregulation, green represents downregulation, and black represents no change in expression. (D) Cluster diagram of miRNAs consistently induced among the four myeloid leukemia cell lines treated with TPA (fold changes $\geq 1.5$; q-value $\leq 5 \%$ ). 

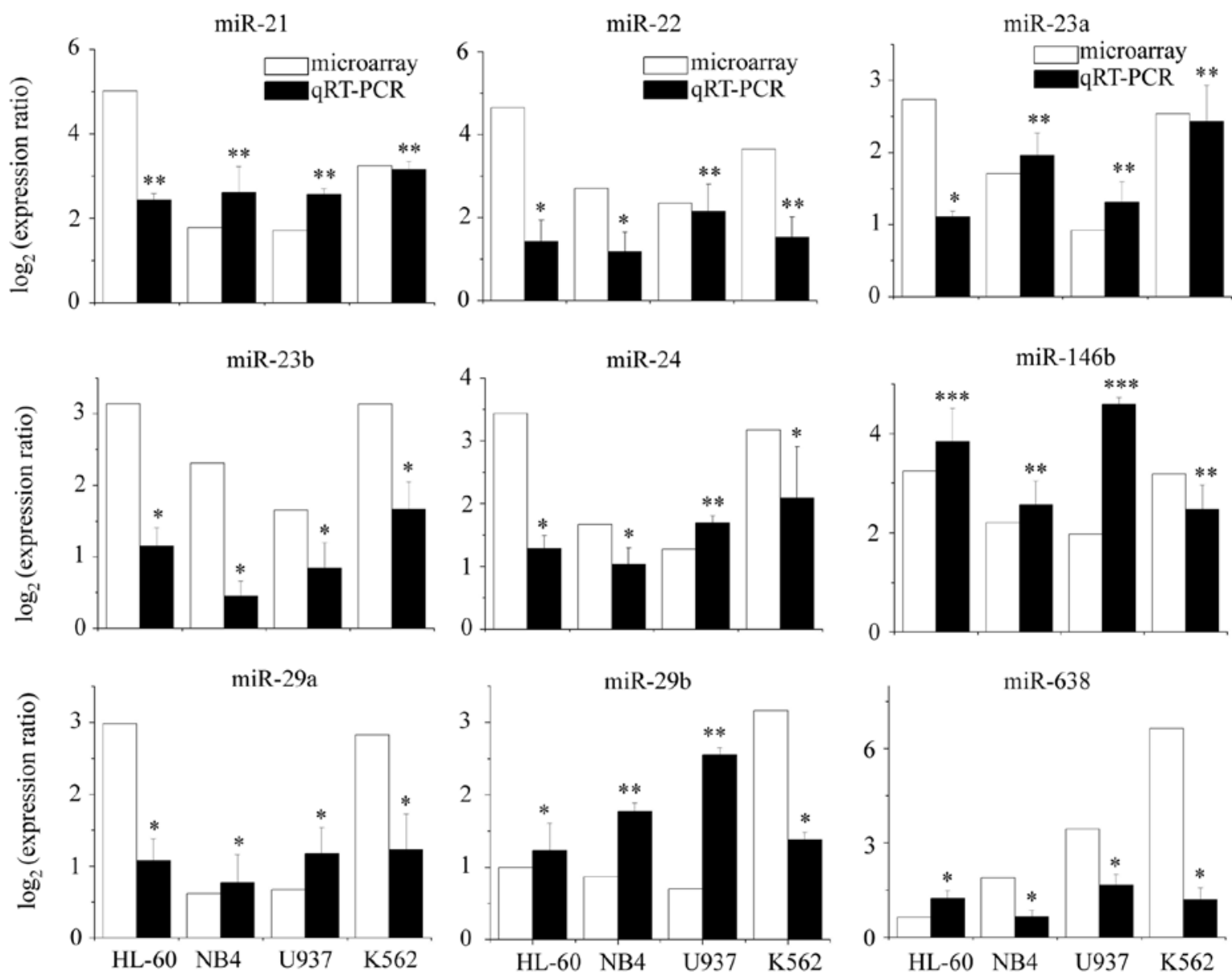

Figure 2. Validation of the microarray results by stem-loop RT-PCR. Stem-loop RT-PCR analysis of nine differentiation-specific miRNAs identified by microarray using independent samples. Data are shown as fold changes of miRNA levels in TPA-treated vs. untreated cells and represent the means \pm SD of three independent experiments. ${ }^{*} \mathrm{P}<0.05,{ }^{* *} \mathrm{P}<0.01$ and ${ }^{* * *} \mathrm{P}<0.001$.

to TPA (Fig. 1D). We support that the differentially regulated miRNAs may represent individual characteristics of each cell line, while the commonly regulated miRNAs may be 'key players' in the differentiation of leukemia cells.

Validation of differentiation-specific miRNAs by quantitative $R T-P C R$. To confirm the accuracy of our microarray analysis, stem-loop RT-PCR assays were performed on the identified differentiation-specific miRNAs (except predicted-miR191) using independent samples. The results of qRT-PCR were consistent with those of microarray analysis in all four cell lines (Fig. 2). These nine miRNAs were confirmed to be differentiation-specific in leukemia cells induced by TPA, of which a subset (miR-21, miR-22, miR-146b, miR-23a and miR-24) was selected for further investigation largely based on the magnitude of the detected induction.

Pharmacological inhibition of MEK/EKR activation suppresses the upregulation of differentiation-specific miRNAs. To ascertain how the expression of these differentiation-specific miRNAs was induced, cell signaling analysis was performed. Both the MEK/ERK and PI3K/Akt pathways are associated with the induction of differentiation by TPA in leukemia cells (11). To investigate the effects of these pathways on miRNA induction, MEK/ERK and PI3K/Akt signal trans- duction was blocked using pharmacological inhibitors: U0126 (MEK1/2 inhibitor) and LY294002 (PI3K inhibitor). U0126 blocked the TPA-stimulated phosphorylation of ERK1/2 (Fig. 3A), and inhibited the induction of miR-21, miR-22, miR-146b, miR-23a and miR-24 (Fig. 3B). The changes in the expression of the differentiation marker, growth arrest, and cell morphology were also inhibited by pretreatment with U0126 (Figs. 3C and 4). By contrast, LY294002 failed to suppress the TPA-induced miRNA expression and cellular differentiation (Figs. 3B, C and 4). The reduction of Akt phosphorylation proved the inhibitory effect of LY294002 on PI3K/ Akt signaling (Fig. 3A). Thus, MEK/ERK activation contributed to the induction of these differentiation-specific miRNAs.

MEK/ERK activation triggers the transcription of differentiation-specific miRNAs. To investigate the mechanism by which MEK/ERK activation caused the upregulation of differentiationspecific miRNAs, the expression of both miRNA biogenesis machineries and primary transcripts was examined. Using immunoblotting analysis, four proteins that are the key machineries of miRNA biogenesis pathways (Drosha, Dicer, Ago1 and Ago2) were investigated. Drosha and Dicer are involved in successively cleaving primary transcripts into mature miRNAs; Agol and Ago2 are the major components of RISC $(5,6)$. The expression of Dicer, Agol and Ago 2 was not significantly altered 

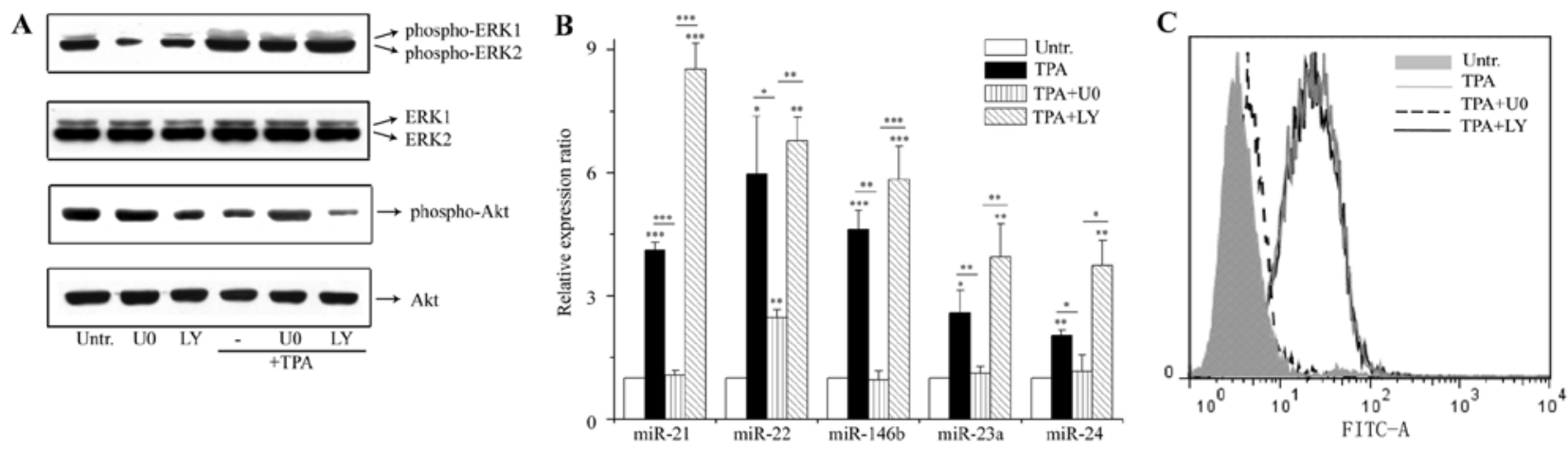

Figure 3. Effects of the MEK1/2 inhibitor U0126 and the PI3K inhibitor LY294002 on the induction of differentiation-specific miRNAs. NB4 cells were pretreated with $10 \mu \mathrm{M} \mathrm{U} 0126$ (U0) or LY294002 (LY) for $30 \mathrm{~min}$ and then treated with $10 \mathrm{nM}$ TPA for $24 \mathrm{~h}$. (A) The levels of ERK, phospho-ERK, Akt and phospho-Akt in cells were analyzed by immunoblotting. The expression levels of total ERK and total Akt were regarded as controls. (B) The induction of miR-21, miR-22, miR-146b, miR-23a and miR-24 was analyzed by qRT-PCR. Data are shown as fold changes of miRNA levels in treated vs. untreated cells and represent the means \pm SD of three independent experiments. ${ }^{*} \mathrm{P}<0.05,{ }^{* * *} \mathrm{P}<0.01$ and ${ }^{* * * *} \mathrm{P}<0.001$ compared to the corresponding control. (C) Flow cytometric analysis of differentiation marker CD14 in cells after different treatments. Results were repeated at least three times, and a typical experimental result is shown.
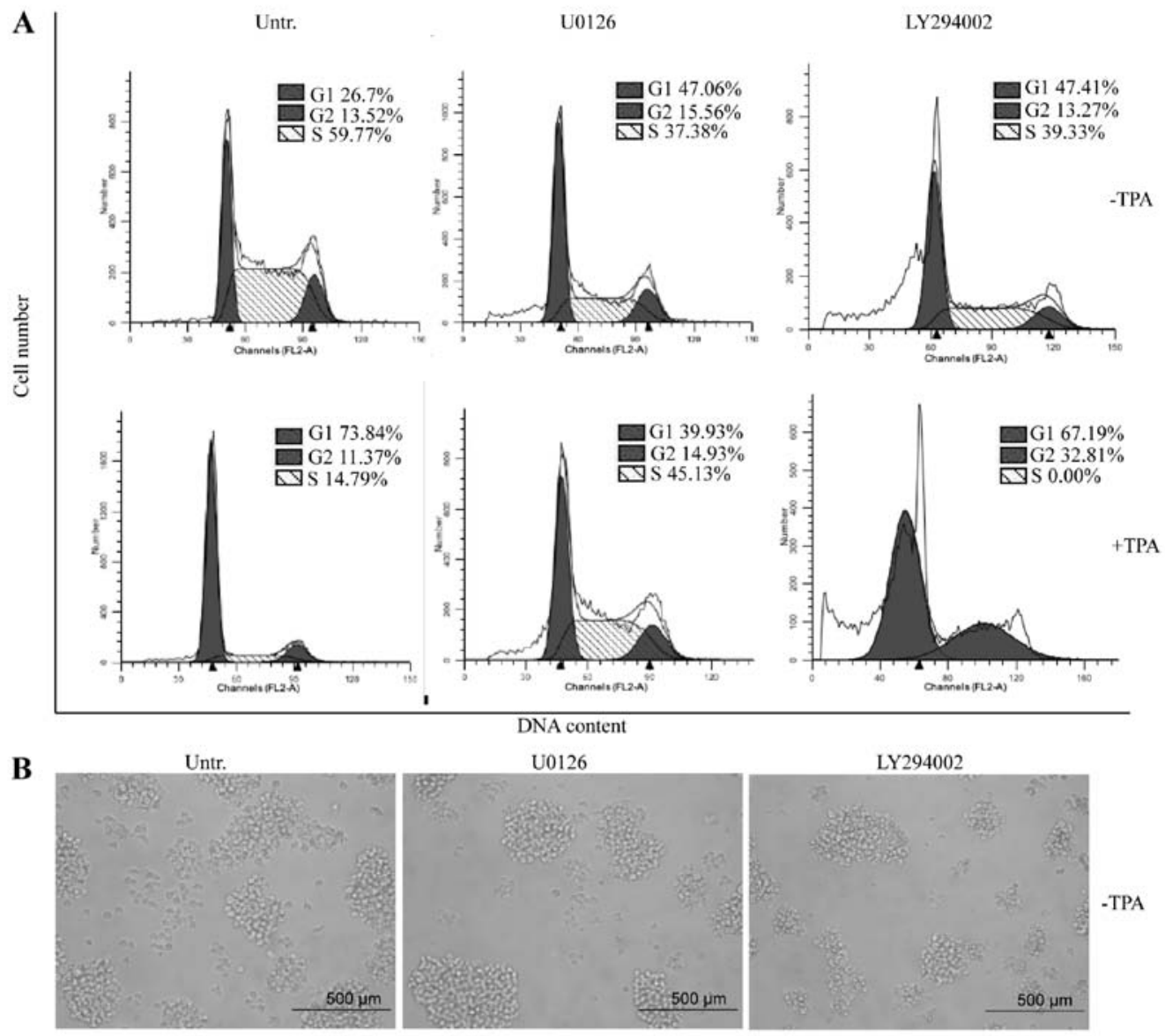

- TPA
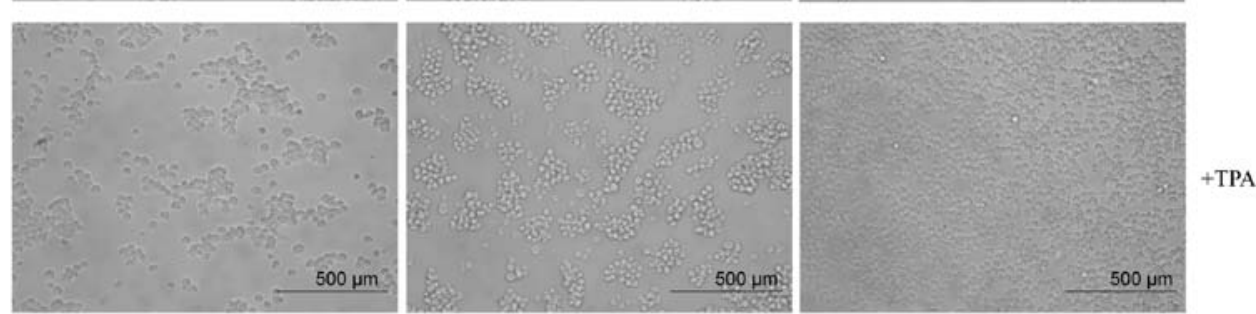

Figure 4. Effects of the MEK1/2 inhibitor U0216 and the PI3K inhibitor LY294002 on the differentiation of leukemia cells induced by TPA. NB4 cells were pretreated with $10 \mu \mathrm{M} \mathrm{U} 0126$ or LY294002 for $30 \mathrm{~min}$ and then treated with $10 \mathrm{nM}$ TPA for $24 \mathrm{~h}$. (A) Flow cytometric analysis of cell cycle distributions. (B) The morphology of cells was observed under light microscopy (scale bar, $500 \mu \mathrm{m}$ ). All experiments were repeated at least three times, and a typical experimental result is shown. 
A

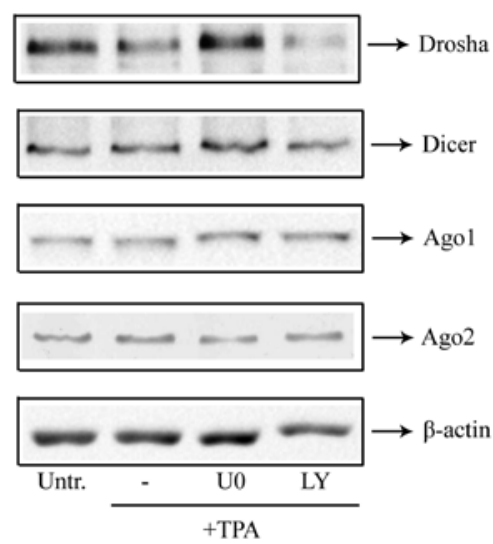

B

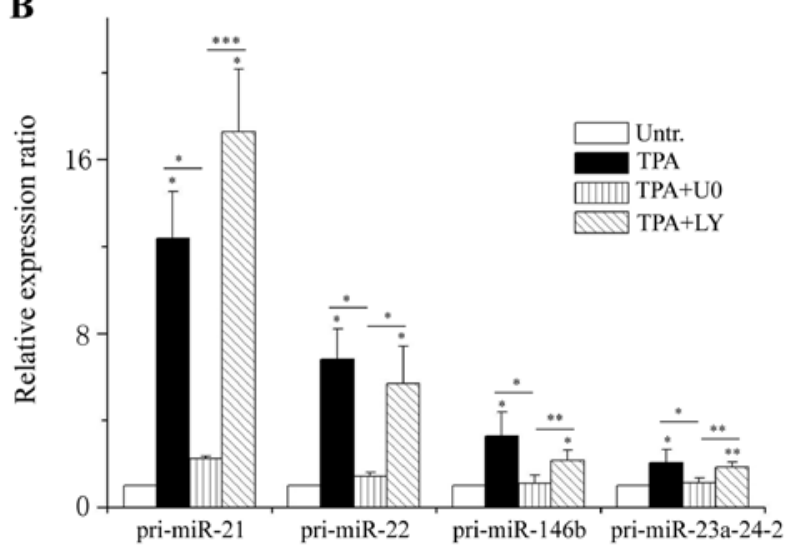

Figure 5. Effects of the MEK1/2 inhibitor U0126 and the PI3K inhibitor LY294002 on the miRNA biogenesis machineries and their transcription. NB4 cells were pretreated with $10 \mu \mathrm{M}$ U0126 (U0) or LY294002 (LY) for $30 \mathrm{~min}$ and then treated with $10 \mathrm{nM}$ TPA for $24 \mathrm{~h}$. (A) The levels of Drosha, Dicer, Ago1 and Ago2 were analyzed by immunoblotting in whole cell lysates. The results are representative of three independent experiments, and $\beta$-actin was used as a loading control. (B) The levels of pri-miR-21, pri-miR-22, pri miR-146b, pri-miR-23a and pri-miR-146b were determined by qRT-PCR Data are shown as fold changes of miRNA levels in treated vs. untreated cells and represent the means $\pm \mathrm{SD}$ of three independent experiments. ${ }^{*} \mathrm{P}<0.05$, ${ }^{* *} \mathrm{P}<0.01$ and ${ }^{* * *} \mathrm{P}<0.001$ compared to the corresponding control.

in differentiated cells. Only Drosha was downregulated in cells after TPA treatment, and U0126 inhibited the downregulation of Drosha caused by TPA (Fig. 5A). These results suggest that the expression levels of the key miRNA biogenesis machineries in differentiated cells were not responsible for the increased expression of the aforementioned miRNAs induced by TPA.

Next, we assessed if the MEK/ERK activation induced the upregulation of the differentiation-specific miRNAs at the transcriptional level. qRT-PCR was performed to examine the primary transcripts of miR-21, miR-22, miR-146b, miR-23a and $\mathrm{miR}-24$. Since there was a coordinated effect of processing at the miR-23a and miR-24 loci (21), a pair of specific primers was designed to quantify the expression levels of the pri-23a24-2 transcript loci. The basal levels of these miRNA primary transcripts were increased after TPA induction, and the induction of these transcripts was significantly inhibited by U0126 but not by LY294002 (Fig. 5B). Therefore, the transcription of these differentiation-specific miRNAs was induced by MEK/ ERK activation, suggesting a major mechanism for the upregulation of these miRNAs in the differentiation of leukemia cells induced by TPA.

\section{Discussion}

This study included: i) miRNA expression (induced by TPA) profiling in four distinct leukemia cell lines; ii) confirmation of the differentiation-specific miRNAs in this process; and iii) investigation of the association of signaling pathways with the induction of differentiation-specific miRNAs, revealing the essential role of MEK/ERK signaling in regulating miRNA transcription in response to TPA treatment.

Since miRNAs are expressed in a tissue-specific and context-dependent manner, the large-scale profiling of miRNAs using microarrays has aided drug research and disease diagnosis (22). Previous studies on miRNA profiling of TPA-treated leukemia cells demonstrated heterogeneous results due to the differences in platform, drug dose, and cell types (7-9). In our study, miRNA expression profiling was analyzed in four distinct leukemia cells using the same experimental platform with the same concentration of drug, which is a better approach for further data mining. Through our comparison, 10 commonly upregulated miRNAs were identified in the four cell lines, representing the differentiation-specific miRNAs. Among them, miR-146b and miR-29a were previously reported to be upregulated in TPA-induced HL-60 cells, and they were also identified in K562 and NB4 cells by our analysis. In addition, miR-23b and miR-24 are induced in K562 cells treated with TPA, and these two miRNAs were also upregulated in TPA-induced U937, NB4 and HL-60 cells by our analysis. Such specifically induced genes that respond to drug treatment are largely regarded as potentially major targets of drug action (20). Therefore, the differentiation-specific miRNAs identified in this study provide significant insights into the thorough understanding of the mechanism of TPA action in leukemia cells.

Signal transduction is an important mechanism for gene regulation in cells (12), and a large number of miRNAs are under the control of various important signal pathways (23). Using specific signal transduction inhibitors, we demonstrated that MEK/ERK activation contributes to the induction of several differentiation-specific miRNAs (miR-21, miR-22, miR-23a, miR-146b and miR-24). These miRNAs target several genes related to differentiation, and most of them function as tumor suppressors (24-26). Their expression patterns correspond to cellular behaviors, i.e., induction in differentiated cells and inhibition in undifferentiated cells. Moreover, these MEK/ ERK signaling-induced miRNAs (such as miR-21 and miR-24) can in turn regulate the MEK/ERK signaling pathway by targeting the components of this pathway or other related pathways, forming a complex regulatory network in TPA-induced leukemia differentiation $(27,28)$. These differentiation-specific miRNAs are an important molecular link between MEK/ERK signal transduction and TPA-induced differentiation.

The common upregulation of differentiation-specific miRNAs via MEK/ERK activation may indicate a uniform regulatory program. To explore this possibility, we examined both miRNA biogenesis machineries and primary transcripts. The expression changes of miRNA biogenesis enzymes can affect the miRNA expression in some cases (29-31). We found that the expression levels of several key miRNA biogenesis machineries were not increased in TPA-induced NB4 cells, and the same trend was also observed in HL-60 and K562 cells (data not shown). Based on this, we hypothesized that MEK/ 
ERK activation may work on the transcriptional level for miRNA production. Indeed, we found that MEK/ERK activation contributed to the induction of the primary transcripts of the differentiation-specific miRNAs by qRT-PCR. Among these transcripts, the upregulation of miR-21 and miR-24 is also observed in other biological processes induced by MEK/ERK activation $(32,33)$. In addition, the promoter regions of these induced miRNAs also contain potential binding sites for the transcription factors RUNX1, NF- $\mathrm{B}$ and RREB-1 $(34,35)$, all of which are downstream effectors of Raf/MEK/ERK signaling. These previous findings and our data indicate a causal effect of the MEK/EKR signaling pathway on the induction of the investigated miRNAs, and further studies will be conducted to confirm the direct targets of the MEK/ERK pathways that contribute to the induction of the differentiation-specific miRNAs.

In conclusion, elucidating the modulation of miRNA expression related to signal transduction advanced our understanding of an intracellular signaling network. Moreover, with increasing clinical administration of differentiation therapy in leukemia patients, the miRNA expression signature reported in this study may facilitate the development of differentiation therapeutic strategies and ultimately be predictive of response to therapy.

\section{Acknowledgements}

We thank Yonggui Wang and Xiaoyu Zhang for their microarray technical assistance, Yu Liu for microarray data analysis, and Chao Wang for flow cytometric analysis. We thank Dr JunWei Guo for the helpful discussion and comments on the manuscript. We also thank Dr M. Bochman for checking the manuscript.

\section{References}

1. Kluiver J, Kroesen BJ, Poppema S and van den Berg A: The role of microRNAs in normal hematopoiesis and hematopoietic malignancies. Leukemia 20: 1931-1936, 2006.

2. Nowak D, Stewart D and Koeffler HP: Differentiation therapy of leukemia: 3 decades of development. Blood 113: 3655-3665, 2009.

3. Schaar D, Goodell L, Aisner J, et al: A phase I clinical trial of 12 O-tetradecanoylphorbol-13-acetate for patients with relapsed/ refractory malignancies. Cancer Chemother Pharmacol 57: 789-795, 2006

4. Chang SH and Hla T: Gene regulation by RNA binding proteins and microRNAs in angiogenesis. Trends Mol Med 17: 650-658, 2011.

5. Kim VN, Han J and Siomi MC: Biogenesis of small RNAs in animals. Nat Rev Mol Cell Biol 10: 126-139, 2009.

6. Davis-Dusenbery BN and Hata A: Mechanisms of control of microRNA biogenesis. J Biochem 148: 381-392, 2010.

7. Kasashima K, Nakamura Y and Kozu T: Altered expression profiles of microRNAs during TPA-induced differentiation of HL-60 cells. Biochem Biophys Res Commun 322: 403-410, 2004.

8. Lal A, Pan Y, Navarro F, et al: miR-24-mediated downregulation of H2AX suppresses DNA repair in terminally differentiated blood cells. Nat Struct Mol Biol 16: 492-498, 2009.

9. Forrest AR, Kanamori-Katayama M, Tomaru Y, et al: Induction of microRNAs, mir-155, mir-222, mir-424 and mir-503, promotes monocytic differentiation through combinatorial regulation. Leukemia 24: 460-466, 2010.

10. Wang XS and Zhang JW: The microRNAs involved in human myeloid differentiation and myelogenous/myeloblastic leukemia. J Cell Mol Med 12: 1445-1455, 2008.

11. Matsumoto E, Hatanaka M, Bohgaki M and Maeda S: PKC pathway and ERK/MAPK pathway are required for induction of cyclin D1 and p21Waf1 during 12-o-tetradecanoylphorbol 13-acetate-induced differentiation of myeloleukemia cells. Kobe J Med Sci 52: 181-194, 2006.
12. Schaar DG, Liu H, Sharma S, et al: 12-O-tetradecanoylphorbol13-acetate (TPA)-induced dual-specificity phosphatase expression and AML cell survival. Leuk Res 29: 1171-1179, 2005.

13. Wang J, Xiang G, Mitchelson K and Zhou Y: Microarray profiling of monocytic differentiation reveals miRNA-mRNA intrinsic correlation. J Cell Biochem 112: 2443-2453, 2011.

14. Kim S, Lee HS, Lee SK, et al: 12-O-Tetradecanoyl phorbol13-acetate (TPA)-induced growth arrest is increased by silibinin by the downregulation of cyclin B1 and cdc2 and the upregulation of p21 expression in MDA-MB231 human breast cancer cells. Phytomedicine 17: 1127-1132, 2010.

15. Park MH, Park SY and Kim Y: Induction of proline-rich tyrosine kinase2 (Pyk2) through C/EBPbeta is involved in PMA-induced monocyte differentiation. FEBS Lett 582: 415-422, 2008.

16. Guo Y, Chen Z, Zhang L, et al: Distinctive microRNA profiles relating to patient survival in esophageal squamous cell carcinoma. Cancer Res 68: 26-33, 2008.

17. Tusher VG, Tibshirani R and Chu G: Significance analysis of microarrays applied to the ionizing radiation response. Proc Natl Acad Sci USA 98: 5116-5121, 2001.

18. Chen C, Ridzon DA, Broomer AJ, et al: Real-time quantification of microRNAs by stem-loop RT-PCR. Nucleic Acids Res 33: e179, 2005.

19. Li X, Liu J, Zhou R, Huang S and Chen XM: Gene silencing of MIR22 in acute lymphoblastic leukaemia involves histone modifications independent of promoter DNA methylation. Br J Haematol 148: 69-79, 2010.

20. Heller G, Schmidt WM, Ziegler B, et al: Genome-wide transcriptional response to 5-aza-2'-deoxycytidine and trichostatin a in multiple myeloma cells. Cancer Res 68: 44-54, 2008.

21. Cameron JE, Fewell C, Yin Q, et al: Epstein-Barr virus growth/ latency III program alters cellular microRNA expression. Virology 382: 257-266, 2008.

22. Nagpal JK, Rani R, Trink B and Saini KS: Targeting miRNAs for drug discovery: a new paradigm. Curr Mol Med 10: 503-510, 2010.

23. Ichimura A, Ruike Y, Terasawa $\mathrm{K}$ and Tsujimoto G: miRNAs and regulation of cell signaling. FEBS J 278: 1610-1618, 2011.

24. Tsuchiya N, Izumiya M, Ogata-Kawata $\mathrm{H}$, et al: Tumor suppressor miR-22 determines p53-dependent cellular fate through posttranscriptional regulation of p21. Cancer Res 71: 4628-4639, 2011.

25. Huang S, He X, Ding J, et al: Upregulation of miR-23a approximately 27 a approximately 24 decreases transforming growth factor-beta-induced tumor-suppressive activities in human hepatocellular carcinoma cells. Int J Cancer 123: 972-978, 2008.

26. Wang P, Zou F, Zhang X, et al: microRNA-21 negatively regulates Cdc25A and cell cycle progression in colon cancer cells. Cancer Res 69: 8157-8165, 2009.

27. Sayed D, Rane S, Lypowy J, et al: MicroRNA-21 targets Sprouty2 and promotes cellular outgrowths. Mol Biol Cell 19: 3272-3282, 2008.

28. Zaidi SK, Dowdy CR, van Wijnen AJ, et al: Altered Runx1 subnuclear targeting enhances myeloid cell proliferation and blocks differentiation by activating a miR-24/MKP-7/MAPK network. Cancer Res 69: 8249-8255, 2009.

29. Lin RJ, Lin YC, Chen J, et al: microRNA signature and expression of Dicer and Drosha can predict prognosis and delineate risk groups in neuroblastoma. Cancer Res 70: 7841-7850, 2010.

30. Vaksman O, Hetland TE, Trope CG, Reich R and Davidson B: Argonaute, Dicer, and Drosha are upregulated along tumor progression in serous ovarian carcinoma. Hum Pathol: May 29, 2012 (Epub ahead of print).

31. Yan M, Huang HY, Wang T, et al: Dysregulated expression of dicer and drosha in breast cancer. Pathol Oncol Res 18: 343-348, 2012.

32. Frezzetti D, De Menna M,Zoppoli P, et al: Upregulation of miR-21 by Ras in vivo and its role in tumor growth. Oncogene 30: 275-286, 2011.

33. Takagi S, Nakajima M, Kida K, Yamaura Y, Fukami T and Yokoi T: MicroRNAs regulate human hepatocyte nuclear factor 4alpha, modulating the expression of metabolic enzymes and cell cycle. J Biol Chem 285: 4415-4422, 2010.

34. Schmeier S, MacPherson CR, Essack M, et al: Deciphering the transcriptional circuitry of microRNA genes expressed during human monocytic differentiation. BMC Genomics 10: 595, 2009.

35. Zhou R, Hu G, Gong AY and Chen XM: Binding of NF-kappaB p65 subunit to the promoter elements is involved in LPS-induced transactivation of miRNA genes in human biliary epithelial cells. Nucleic Acids Res 38: 3222-3232, 2010. 